\title{
Simple proofs of Steck's determinantal expressions for probabilities in the Kolmogorov and Smirnov tests
}

\section{E.J.G. Pitman}

This paper gives simple proofs of two theorems of Steck concerning the distribution of sample distribution functions.

Theorems I and II below were stated and proved by Steck in two notable papers [2], [3]. As Steck showed, Theorem I enables us to determine:

(i) the probability that the empirical distribution function lies between two other distribution functions;

(ii) very general confidence regions for an unknown distribution function;

(iii) the power of a test based on the empirical distribution function.

From Theorem II we can obtain the null distribution of the two-sample Smirnov statistics for arbitrary sample sizes. Steck's proofs were indirect, and somewhat complicated. Mohanty [1] gave a shorter proof of Theorem II. Here I give simple, direct proofs of both theorems.

LEMMA. Let

$$
\begin{aligned}
& A_{1}, A_{2}, \ldots, A_{m-1}, \\
& B_{1}, B_{2}, \ldots, B_{m}
\end{aligned}
$$

denote events such that for any integer $k$, the sets

Received 28 April 1972. 


$$
\left\{A_{r}, B_{s} ; r<k, s \leq k\right\},\left\{A_{r}, B_{s} ; r>k, s>k\right\}
$$

are independent, then

$$
P\left(B_{1} B_{2} \cdots B_{m} A_{1} A_{2} \cdots A_{m-1}\right)=\Delta_{m}=\operatorname{det}\left(d_{i j}\right), \quad 1 \leq i, j \leq m,
$$

where

$$
\begin{array}{rlrl}
d_{i j} & =0 & & \text { if } i>j+1, \\
& =1 & & \text { if } i=j+1, \\
& =P\left(B_{i}\right) & & \text { if } i=j, \\
& =P\left(B_{i} B_{i+1} \cdots B_{j} A_{i}^{*} A_{i+1}^{*} \cdots A_{j-1}^{*}\right) & \text { if } i<j,
\end{array}
$$

and $A_{r}^{*}$ is the complement of $A_{r}$.

Note that the conditions on the events make $B_{1}, B_{2}, \ldots, B_{m}$ independent. The events $A_{1}, A_{2}, \ldots$ are 1-dependent. Put

$$
\bar{A}_{r}=A_{1} A_{2} \cdots A_{r}, \bar{B}_{r}=B_{1} B_{2} \cdots B_{r} \text {. }
$$

The lemma may be proved by use of the principle of inclusion and exclusion.

$$
\begin{aligned}
P\left(\bar{B}_{m} A_{1} A_{2} \cdots A_{m-1}\right)=P\left(\bar{B}_{m}\right)-\sum P\left(\bar{B}_{m} A_{r}^{*}\right) & +\sum_{r<s} P\left(\bar{B}_{m} A_{r}^{*} A_{s}^{*}\right) \\
& -\ldots+(-1)^{m-1} P\left(\bar{B}_{m} A_{1}^{*} A_{2}^{*} \ldots A_{m-1}^{*}\right),
\end{aligned}
$$

which can be shown directly to be the expansion of $\Delta_{m}$. A proof by induction is shorter, and easier to print.

Assume the lemma true for $m=n$.

$$
\Delta_{n}=P\left(B_{n} \bar{A}_{n-1}\right)
$$

Consider $\Delta_{n+1}$. The elements of its last row are all zero except

$$
a_{n+1, n}=1, d_{n+1, n+1}=P\left(B_{n+1}\right) \text {. }
$$

Therefore

$$
\Delta_{n+1}=P\left(B_{n+1}\right) \Delta_{n}-\Delta_{n}^{\prime}
$$

where $\Delta_{n}^{\prime}$ differs from $\Delta_{n}$ only in having $B_{n}$ in the last column of $\Delta_{n}$ 
replaced by ${ }_{n}^{B}{ }_{n+1} A_{n}^{*}$, which satisfies the same conditions relative to the other events appearing in $\Delta_{n}^{\prime}$ as does $B_{n}$. Therefore

$$
\begin{gathered}
\Delta_{n}^{\prime}=P\left(\bar{B}_{n} B_{n+1} A_{n}^{*} \bar{A}_{n-1}\right)=P\left(\bar{B}_{n+1} \bar{A}_{n-1} A_{n}^{*}\right) ; \\
P\left(B_{n+1}\right) \Delta_{n}=P\left(B_{n+1}\right) P\left(\bar{B}_{n} \bar{A}_{n-1}\right)=P\left(\bar{B}_{n+1} \bar{A}_{n-1}\right) .
\end{gathered}
$$

Thus

$$
\begin{aligned}
\Delta_{n+1} & =P\left(\bar{B}_{n+1} \bar{A}_{n-1}\right)-P\left(\bar{B}_{n+1} \bar{A}_{n-1} A_{n}^{*}\right) \\
& =P\left(\bar{B}_{n+1} \bar{A}_{n-1} A_{n}\right)=P\left(\bar{B}_{n+1} \bar{A}_{n}\right),
\end{aligned}
$$

and so the lemma is true for $m=n+1$. It is easy to show that it is true for $m=2$, and so it is true for all $m$.

COROLLARY. Taking the case where every $P\left(B_{i}\right)=1$, we obtain the following result for the 1-dependent sequence of events $A_{1}, A_{2}, \ldots$,

$$
P\left(A_{1} A_{2} \cdots A_{m-1}\right)=\operatorname{det}\left(d_{i j}\right),
$$

where

$$
\begin{array}{rlrl}
d_{i j} & =0 & & \text { if } i>j+1, \\
& =1 & & \text { if } i=j \text { or } j+1, \\
& =P\left(A_{i}^{*} A_{i+1}^{*} \cdots A_{j-1}^{*}\right) & \text { if } i<j .
\end{array}
$$

THEOREM I. Let

$$
\begin{aligned}
& 0 \leq u_{1} \leq u_{2} \leq \ldots \leq u_{m} \leq 1, \\
& 0 \leq v_{1} \leq v_{2} \leq \ldots \leq v_{m} \leq 1,
\end{aligned}
$$

be given constants such that

$$
u_{i}<v_{i}, i=1,2, \ldots, m \text {. }
$$

If $U_{1}, U_{2}, \ldots, U_{m}$ are the order etatistics (in ascending order) from a sample of $m$ independent uniform random variables with range 0 to 1 ,

$$
P\left(u_{i} \leq U_{i} \leq v_{i}, 1 \leq i \leq m\right)=m ! \operatorname{det}\left[\left(v_{i}-u_{j}\right)_{+}^{j-i+1} /(j-i+1) !\right],
$$

where $(x)_{+}=\max (x, 0)$, and it is understood that determinont elements 
for which $i>j+1$ are all zero.

Proof. Let $Y_{1}, Y_{2}, \ldots, Y_{m}$ be independent random variables, each with a uniform distribution from 0 to 1 . The required probability is equal to

$$
m ! P\left(u_{i} \leq y_{i} \leq v_{i}, 1 \leq i \leq m ; y_{1} \leq y_{2} \leq \ldots \leq y_{m}\right)
$$

Denote by $B_{i}$ the event $u_{i} \leq Y_{i} \leq v_{i}$. Denote by $A_{i}$ the event $Y_{i} \leq Y_{i+1}$, and by $A_{i}^{*}$ the complement of $A_{i}$, that is, the event $y_{i}>Y_{i+1}$. The events $A_{i}, B_{i}$ satisfy the conditions of the lemma. Hence

(2) $P\left(u_{i} \leq y_{i} \leq v_{i}, 1 \leq i \leq m ; y_{1} \leq y_{2} \leq \ldots \leq y_{m}\right)$

$$
\begin{aligned}
& =P\left(B_{1} B_{2} \cdots B_{m} A_{1} A_{2} \cdots A_{m-1}\right)=\operatorname{det}\left(d_{i j}\right) . \\
d_{i i}= & P\left(B_{i}\right)=v_{i}-u_{i} .
\end{aligned}
$$

If $i<j, d_{i j}=P\left(B_{i} B_{i+1} \cdots B_{j} A_{i}^{*} A_{i+1}^{*} \cdots A_{j-1}^{*}\right)$. The event $B_{i} B_{i+1} \cdots B_{j} A_{i}^{*} A_{i+1}^{*} \cdots A_{j-1}^{*}$ is

$$
\begin{aligned}
& u_{r} \leq y_{r} \leq v_{r}, i \leq r \leq j, \\
& y_{i}>y_{i+1}>\ldots>y_{j} .
\end{aligned}
$$

This is equivalent to

$$
v_{i} \geq y_{i}>y_{i+1}>\ldots>y_{j} \geq u_{j},
$$

the probability of which is $\left(v_{i}-u_{j}\right)_{+}^{j-i+1} /(j-i+1) !$. The theorem then follows from (1) and (2).

THEOREM II. Let $b_{1} \leq b_{2} \leq \ldots \leq b_{m}$ and $c_{1} \leq c_{2} \leq \ldots \leq c_{m}$ be sequences of integers such that $b_{i}<c_{i}$. The number of sets of integers $\left(R_{1}, R_{2}, \cdots, R_{m}\right)$ such that

$$
\begin{aligned}
& R_{1}<R_{2}<\ldots<R_{m}, \\
& b_{i}<R_{i}<c_{i}, \quad 1 \leq i \leq m
\end{aligned}
$$


is the m-th order determinant $\operatorname{det}\left(d_{i j}\right)$, where

$$
\begin{aligned}
d_{i j} & =0 & \text { if } i>j+1 \text { or if } c_{i}-b_{j} \leq 1, \\
& =\left(\begin{array}{c}
c_{i}-b_{j}+j-i-1 \\
j-i+1
\end{array}\right) & \text { otherwise. }
\end{aligned}
$$

Proof. Put $y_{i}=R_{i}-i, u_{i}=b_{i}-i+1, v_{i}=c_{i}-i-1$. The conditions on the $R_{i}$ are equivalent to

$$
\begin{gathered}
y_{i} \text { an integer, } u_{i} \leq Y_{i} \leq v_{i} ; 1 \leq i \leq m, \\
y_{1} \leq Y_{2} \leq \ldots \leq y_{m} .
\end{gathered}
$$

As before, denote by $A_{i}$ the event $Y_{i} \leq Y_{i+1}$, and by $A_{i}^{*}$ its complement, the event $y_{i}>y_{i+1}$. Put $N_{i}=v_{i}-u_{i}+1$.

The required number is equal to

$$
N_{1} N_{2} \cdots N_{m} P\left(A_{1} A_{2} \cdots A_{m-1}\right)
$$

when the $Y_{i}$ are independent random variables, and $Y_{i}$ has a uniform distribution over the integers from $u_{i}$ to $v_{i}$. By the corollary to the lemma, this is

$$
N_{1} N_{2} \cdots N_{m} \operatorname{det}\left(d_{i j}^{\prime}\right)
$$

where

$$
\begin{array}{rlrl}
d_{i j}^{\prime} & =0 & & \text { if } i>j+1, \\
& =1 & \text { if } i=j \text { or } j+1, \\
& =P\left(A_{i}^{*} A_{i+1}^{*} \ldots A_{j-1}^{*}\right) & \text { if } i<j ; \\
P\left(A_{i}^{*} A_{i+1}^{*} \ldots A_{j-1}^{*}\right) & =P\left(v_{i} \geq Y_{i}>y_{i+1}>\ldots>y_{j} \geq u_{j}\right)
\end{array}
$$

as before. This is zero if $v_{i}-u_{j}<j-i$, that is if $c_{i}-b_{j} \leq 1$. Otherwise it is equal to

$$
\left(\begin{array}{c}
v_{i}^{-u_{j}+1} \\
j-i+1
\end{array}\right) / N_{i} N_{i+1} \cdots N_{j}=\frac{d_{i j}}{N_{i} N_{i+1} \cdots N_{j}} .
$$


The numerator is the number of vectors of integers $\left(y_{i}, y_{i+1}, \ldots, y_{j}\right)$ satisfying $v_{i} \geq y_{i}>y_{i+1}>\ldots>y_{j} \geq u_{j}$, and the denominator is the number of vectors satisfying $u_{r} \leq y_{p} \leq v_{p}, i \leq r \leq j$.

Put $M_{0}=1, M_{r}=N_{1} N_{2} \cdots N_{r}$; then in all cases

$$
d_{i j}^{\prime}=d_{i j} M_{i-1} / M_{j}
$$

The required number (3) is

$$
M_{m} \operatorname{det}\left(d_{i j}^{\prime}\right)=M_{m} \operatorname{det}\left(d_{i j} M_{i-1} / M_{j}\right)=\operatorname{det}\left(d_{i j}\right) \text {, }
$$

as may be obtained by taking factors out of rows and out of columns. This proves the theorem.

\section{References}

[1] S.G. Mohanty, "A short proof of Steck's result on two-sample Smirnov statistics", Ann. Math. Statist. 42 (1971), 413-414.

[2] G.P. Steck, "The Smirnov two sample tests as rank tests", Ann. Math. Statist. 40 (1969), 1449-1466.

[3] G.P. Steck, "Rectangle probabilities for uniform order statistics and the probability that the empirical distribution function lies between two distribution functions", Ann. Math. Statist. 42 (1971), 1-11.

301 Davey Street,

Hobart,

Tasmania 7000 . 